\title{
STUDY OF CHANGES IN SERUM LEVELS OF CREATINE KINASE AND LIVER ENZYMES IN ORGANOPHOSPHORUS COMPOUND POISONING AND IT'S PROGNOSTIC SIGNIFICANCE IN CHENGALPATTU MEDICAL COLLEGE HOSPITAL
}

\author{
Velure Rajarao Mohan Rao', Arjunan Senthilkumaran'²
}

1 Professor, Department of Medicine, Chengalpattu Medical College and Hospital.

2Junior Resident, Department of Medicine, Chengalpattu Medical College and Hospital.

ABSTRACT

Organophosphorus compound poisoning is one of the most common poisoning in rural areas. The main symptoms of organophosphorus compound poisoning is due to stimulation of nicotinic and muscuranic receptors. The cholinergic symptoms and prognosis is assessed by acetylcholinesterase levels, which is not readily available in all clinical laboratories. So, a cheaper and easily quantifiable serum markers like creatine kinase and liver enzymes can be used in predicting as well as assessing the prognosis with organophosphorus compound poisoning. A prospective study was done in about 110 patients with consumption of organophosphorus compound poisoning admitted in the department of medicine, Chengalpattu Medical College and Hospital. Patients with history of chronic liver disease and myopathy were excluded. Patients were classified according to peradeniya organophosphorus poisoning scale. The present study found that the initial serum CPK level is comparable for BChE level and can be used as an alternative biomarker in diagnosis of acute organophosphorus compound poisoning provided that exclusion of any other diseases or conditions that may cause rise in CPK levels, these results were statistically significant $(p<0.05)$.

\section{KEYWORDS}

Acetylcholinesterase, Peradeniya Poisoning Scale, Creatine Kinase, Liver Enzymes.

HOW TO CITE THIS ARTICLE: Rao VRM, Senthilkumaran A. Study of changes in serum levels of creatine kinase and liver enzymes in organophosphorus compound poisoning and it's prognostic significance in Chengalpattu medical college hospital. J. Evolution Med. Dent. Sci. 2016;5(63):4419-4422, DOI: 10.14260/jemds/2016/1010

\section{INTRODUCTION}

Dr. Kuntal Bhattacharyya. ${ }^{1}$ did a prospective study in 2011 in the Department of General Medicine, Government Medical College, Kolkata, India, studied 63 patients of organophosphorus compound poisoning to find out the level of creatine kinase and its prognostic significance. The serum creatine kinase level was estimated for 61 patients. Two patients (Initial CPK 1138 IU/L and 1086 IU/L) collapsed out of complications during the course of therapy on day 3 and day 5 respectively. It was found that mortality was more in patients with high initial CPK level. Another 3 patients developed intermediate syndrome on day 3 had initial creatine kinase level $1138 \mathrm{IU} / \mathrm{L}$ and concluded serum creatine kinase can be an efficient biomarker in case of acute organophosphorus poisoning.

S. B. Agarwal. ${ }^{2}$ in the year 2007 conducted a prospective study in acute OPC poisoning patients treated in the Inpatient Department of General Medicine, B.J.M.C.H, Ahmedabad. 121 patients were studied and found that the levels of serum LDH and creatine kinase levels were markedly elevated among poisoning cases indicating muscular impairment due to organophosphorus compound toxicity. The creatine kinase level was markedly increased among poison cases and there was high elevation in patients who collapsed. A. Patel, V. Shivgotra, and V. Bhatnagar. ${ }^{3}$ in the year 2008 conducted a prospective study in workers engaged in organophosphorus insecticide production.

Financial or Other, Competing Interest: None.

Submission 30-06-2016, Peer Review 21-07-2016,

Acceptance 30-07-2016, Published 05-08-2016.

Corresponding Author:

Velure Rajarao Mohan Rao,

Professor of Medicine,

Department of Medicine,

Chengalpattu Medical College.

E-mail:vrmohan_rao@yahoo.co.in

DOI: $10.14260 /$ jemds/2016/1010
About 161 workers were involved as subjects in the study. There was 40 subjects in control group, 50 subjects in maintenance group, and 71 subjects in exposed group. The serum levels of SGOT and SGPT was in the normal level in the controlled and in the subjects who were exposed.

Antonio F. Hernandez ${ }^{4}$ in the year 2006 conducted a cohort study involving more than 100 intensive farmers. The renal and liver parameters like urea, creatinine, SGOT, SGPT, and LDH was tested twice during the time for spraying. Cholinesterase suppression by more than $25 \%$ was used as criteria for estimating insecticide exposure. The results showed a correlation with increase in SGOT, SGPT, and LDH levels.

Dilshad Khan, Mahwish Bhatti, Farooq Khan, Syed Naqvi, Karam A. ${ }^{5}$ pathologists in Army Medical College, in 2008, conducted a prospective study on 109 patients and concluded that butryl cholinesterase level was significantly $(\mathrm{P}<0.001)$ reduced in the farmers exposed to insecticide when compared to controls. Biochemical parameters like SGOT, SGPT, creatine phosphokinase, lactate dehydrogenase were markedly raised in the insecticide exposed agriculture workers as compared to control group. Total insecticide residues showed a highly significant correlation with SGOT, LDH, SGPT.

The major toxicity of organophosphorus compound poisoning is due to inhibition of acetylcholinesterase, which leads to stimulation of muscuranic and nicotinic receptors. Cholinergic symptoms develop fast, which forms the basis for clinical diagnosis, which is confirmed by cholinesterase inhibition. Cholinesterase levels are not routinely done in all clinical labs and is not readily available in many parts of india, where clinical case load is very high. In Chengalpattu Medical College, around 1200 cases per annum get admitted with organophosphorus compound poisoning. A cheaper, easily quantifiable, and easily available biochemical markers in relation to organophosphorus compound poisoning like serum CPK and liver enzymes can be used in predicting as well 
as assessing the prognosis with organophosphorus compound poisoning.

\section{MATERIAL AND METHODS}

The study was conducted during the period of November 2014-October 2015 in cases admitted in the Department of Medicine, Chengalpattu Medical College. About 110 patients with consumption of organophosphorus compound poisoning admitted in Department of Medicine, Chengalpattu Medical College were included in the study. Study design is a prospective study. Patients with history of chronic liver disease and myopathy were excluded. Patients included in the study were classified according to Peradeniya organophosphorus poisoning scale into mild (0-3), moderate (4-7), and severe (8-11).

\begin{tabular}{|llc|}
\hline \hline Parameter & Criteria & Score \\
\hline Pupil size & $\geq 2 \mathrm{~mm}$ & 0 \\
& $<2 \mathrm{~mm}$ & 1 \\
& Pinpoint & 2 \\
Respiratory rate & $<20 / \mathrm{min}$ & 0 \\
& $\geq 20 / \mathrm{min}$ & 1 \\
& $\geq 20 / \mathrm{min}$ with central cyanosis & 2 \\
Heart rate & $>60 / \mathrm{min}$ & 0 \\
& $41-60 / \mathrm{min}$ & 1 \\
& $<40 / \mathrm{min}$ & 2 \\
Fasciculation & None & 0 \\
& Present, generalized/continuous & 1 \\
& Both generalized and continuous & 2 \\
Level of consciousness & Conscious and rationale & 0 \\
& Impaired response to verbal commands & 1 \\
Seizures & No response to verbal commands & 2 \\
& Absent & 0 \\
\hline 0-3: mild poisoning; 4-7: moderate poisoning; 8-II: severe poisoning. & 1 \\
POP: Peradeniya Organophosphorus Poisoning & \\
\hline
\end{tabular}

\section{RESULTS}

In the studied group of 110 patients with organophosphorus compound poisoning brought to Chengalpattu Medical College Hospital, $62.72 \%$ were males and $37.27 \%$ were females. Majority of the patients were in the age group of 20-40, which constituted $61 \%$ of the study population. The severe poisoning group predominantly belonged to older population with a mean age 51.48 years when compared to mild 36 years and moderate 32.02 years. In the studied population, $22(20 \%)$ had mild, 47 (42.72\%) had moderate, and 41 (37.27\%) had severe level of poisoning. Weissmann-Brenner et $\mathrm{al}^{6}$ reported that $66 \%$ of patients with OP poisonings were males and $34 \%$ were females, $39 \%$ were less than 10 years old, $64 \%$ of exposure was accidental, $36 \%$ was suicidal and the most common route of intoxication was oral (67\%). Most common compound consumed was chlorpyrifos (32.72\%) followed by methylparathion (19.09\%) and dimethoate (17.27\%). Most common presenting symptom is abdominal pain (86.36\%) and most common presenting sign is miosis (89.09\%).

Mean CPK, SGOT, and SGPT values in case of mild OPC poisoning are $76.5,38.63$, and 45.0 respectively. Mean CPK, SGOT, and SGPT values in case of moderate severity in case of OPC poisoning are $249.24,71.53$, and 70.44 respectively. Mean CPK, SGOT, and SGPT values in case of severe poisoning are 843.68, 157.54, and 138.32 respectively. Mean CPK, SGOT, SGPT values in case of respiratory failure, intermediate syndrome, and death are $(815.10,157.84,141.57),(857.2$, $159.4,143.40),(940.81,183.37,160.37)$ respectively.
Association of Severe Organophosphorus Compound Poisoning by Peradeniya Organophosphorus Poisoning Scale with CPK, SGOT, and SGPT

\begin{tabular}{|c|c|c|c|}
\hline Severe Poisoning & CPK & SGOT & SGPT \\
\hline Mean & 843.68 & 157.54 & 138.32 \\
\hline $\mathrm{N}$ & 41 & 41 & 41 \\
\hline SD & 13.0205 & 52.7101 & 49.1322 \\
\hline Minimum & 564.50 & 32.00 & 33.00 \\
\hline Maximum & 1100.00 & 301.00 & 288.00 \\
\hline Range & 535.50 & 269.00 & 255.00 \\
\hline
\end{tabular}

Association of Respiratory Failure in Organophosphorus Poisoning with CPK, SGOT, and SGPT

\begin{tabular}{|ll|c|c|c|}
\hline PROGNOSIS & & CPK & SGOT & SGPT \\
\hline Respiratory & Mean & 815.1053 & 157.8421 & 141.5789 \\
failure & $\mathrm{N}$ & 19 & 19 & 19 \\
& Std. & 223.841 & 40.23578 & 36.19441 \\
& Deviation & & & \\
& Minimum & 241.50 & 67.00 & 45.00 \\
& Maximum & 1100.00 & 211.00 & 198.00 \\
& Range & 858.50 & 144.00 & 153.00 \\
\hline
\end{tabular}

Association of Intermediate Syndrome in Organophosphorus Poisoning with CPK, SGOT, and SGPT

\begin{tabular}{|ll|c|c|c|}
\hline Intermediate & Mean & 857.2000 & 159.4000 & 143.4000 \\
syndrome & $\mathrm{N}$ & 5 & 5 & 5 \\
& Std. & 74.99133 & 39.75299 & 35.76031 \\
& Deviation & & 98.00 & 86.00 \\
& Minimum & 770.00 & 198.00 & 176.00 \\
& Maximum & 955.00 & 100.00 & 90.00 \\
\hline
\end{tabular}

Association of Death in Organophosphorus Poisoning with CPK, SGOT, and SGPT

\begin{tabular}{|ll|c|c|c|}
\hline death & Mean & 940.8125 & 185.3750 & 160.3750 \\
& $\mathrm{~N}$ & 8 & 8 & 8 \\
& Std. & 108.348 & 24.07095 & 25.24134 \\
& Deviation & & & \\
& Minimum & 788.50 & 156.00 & 135.00 \\
& Maximum & 1100.00 & 211.00 & 198.00 \\
& Range & 311.50 & 55.00 & 63.00 \\
\hline
\end{tabular}

Association of Mild Severity by Peradeniya Score of Organophosphorus (POP) with CPK, SGOT, and SGPT

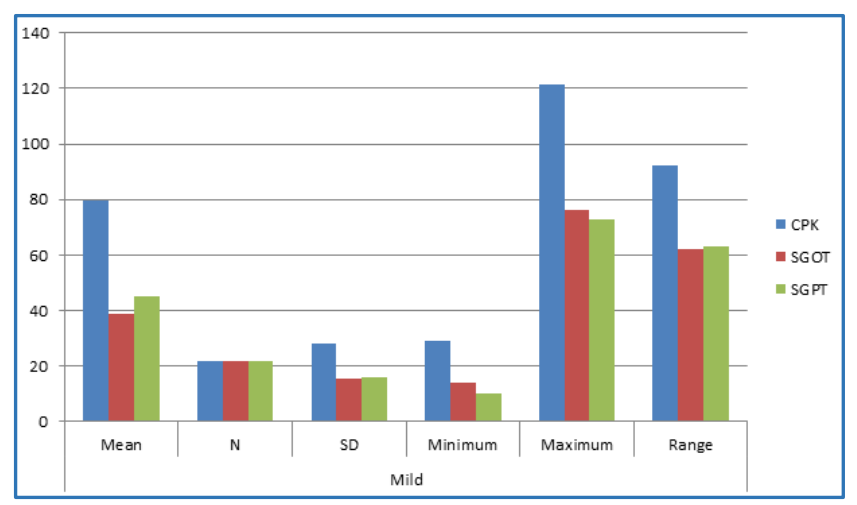


Association of Moderate Severity by Peradeniya score of Organophosphorus (POP) with CPK, SGOT, and SGPT

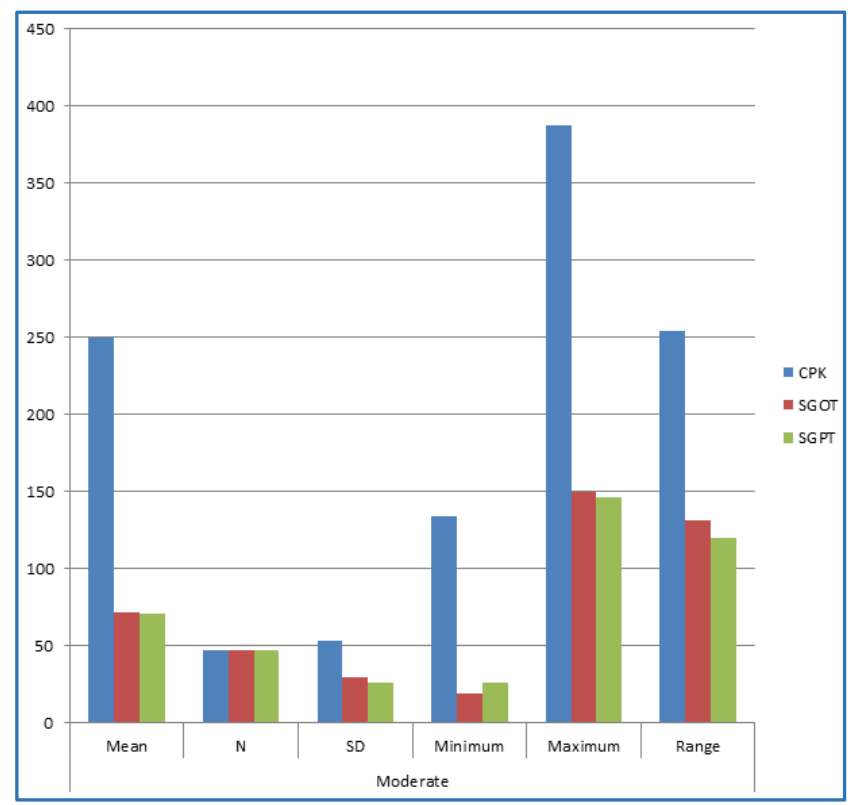

Association of Severe Severity by Peradeniya Score of Organophosphorus (POP) with CPK, SGOT, and SGPT

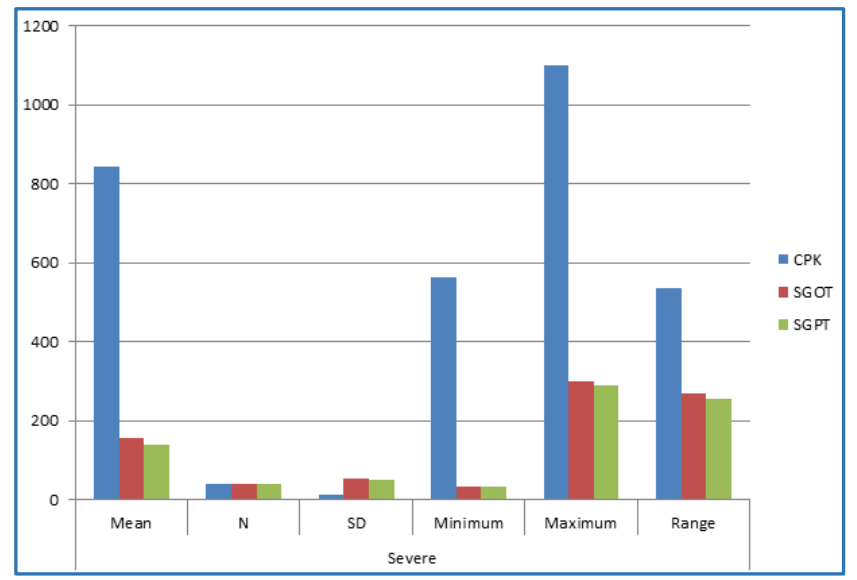

\section{DISCUSSION AND CONCLUSION}

The present study found that the initial serum CPK level is comparable for BChE level and can be used as an alternative biomarker in diagnosis of acute organophosphorus compound poisoning provided that exclusion of any other diseases or conditions that may cause rise in CPK levels, these results were statistically significant $(\mathrm{p}<0.05)$. This was in agreement with Perreault et $\mathrm{al}^{7}$ who confirmed that when a skeletal muscle is injured, CPK leaks into the blood and urine. Serum CPK level remains the best biomarker for detecting and monitoring skeletal muscle damage and diseases. Also, authors confirmed the elevation of serum CPK levels in acute organophosphorus compound poisoning especially if the patient is severely poisoned presumably due to muscle fiber necrosis.

Various authors illustrated that the POP scale can efficiently predict the severity of organophosphorus compound poisoned patients. Muscle injury in insecticide poisoning occurs in three types. Type 1 is because of continuous depolarisation at NM junction, type 2 is intermediate syndrome and type 3 is delayed polyneuropathy.
In the present work, the elevated serum CPK levels were confirmed during the acute stage of toxicity i.e. all cases presented within 6 hours of exposure to organophosphorus compounds and before the development of the intermediate syndrome.

This was in agreement with authors who confirmed in their study on OP intoxicated patients that serum CPK level is elevated even in the absence of intermediate syndrome presumably due to muscle fiber necrosis. Intermediate syndrome occurs in between the periods of acute and delayed organophosphorus toxicity. In majority of cases, intermediate syndrome occurs in between 24-72 hours after acute organophosphorus poisoning. Meanwhile, authors had linked the raised CPK levels to the rhabdomyolysis in intermediate syndrome. The excess acetylcholine seen in OP poisoning leads to reversible myocyte injury and rise of different muscle enzymes including CPK. The present work highlighted the importance of measurement of serum CPK levels as it might be helpful in predicting as well as assessing the prognosis of patients with acute organophosphorus compound poisoning.

These results are in agreement with Bhattacharyya et al ${ }^{2}$ who confirmed the presence of a high degree of correlation between initial CPK value and POP scale. Muscle fiber necrosis and consequently raised CPK levels occur in severely acute organophosphorus poisoned cases. So, cheaper, easily quantifiable, and more available biochemical markers in relation to OP poisoning like serum CPK can be used in predicting as well as assessing the prognosis of patients with organophosphorus compound poisoning.

However, the main disadvantage of serum CPK as a biomarker for acute organophosphorus compound poisoning, its nonspecificity. So, exclusion of other conditions and diseases that may cause its elevation in patients with acute organophosphorus compound poisoning is mandatory. Sniderman et al ${ }^{8}$ stated that numerous factors have influence on CPK activity, so the suitability of CPK as a biomarker for diagnosis of muscle injury and disease should be viewed with caution. Also, researches illustrated that there are multiple causes of elevated CPK, which may affect its reliability as a biomarker. Also, further studies with greater number of patients and in other locations are required to support the present observations since the present work was conducted on a relatively small number of patients and in only one poisoning control unit with most of cases were presented with moderate organophosphorus toxicity and CPK levels are commonly elevated in severely intoxicated patients.

Liver accepts and metabolises organophosphates through oxidation and sulfate or glucuronate conjugation and may undergo oxidative damage in case of organophosphorus poisoning. Hence, liver function test like SGOT and SGPT values are raised in case severe organophosphorus poisning.

In this study, SGOT when compared to SGPT is abnormal in more case of organophosphorus poisoning and their increase depends on type of compound consumed. Main limitation of liver function test is underlying silent chronic liver disease cannot be ruled out and its nonspecificity.

Limitation of the study are sample size was small. The amount of toxin ingested and time lapsed after consumption of the poison had not been considered. An underlying chronic liver disease and myopathy cannot be ruled out, which might have altered the results. 


\section{REFERENCES}

1. Bhattacharyya K, Phaujdar S, Sarkar R, et al. Serum creatine kinase: a probable marker of severity in organophosphorus poisoning. Toxicol Int 2011;18(2): 117-23.

2. Agarwal SB, Bhatnagar V, Agarwal A, et al. Impairment in clinical indices in acute organophosphate insecticide poisoning patients in India. The Internet Journal of Toxicology 2007;4(1).

3. Patel AV, Shivgotra VK, Bhatnagar VK. Biochemical indices in workers engaged in production and formulation of organophosphate insecticides. The Internet Journal of Toxicology 2008;5(2).
4. Khan DA, Bhatti MM, Khan FA, et al. Adverse effects of pesticides residues on biochemical markers in pakistani tobacco markers. Int J Clin Exp Med 2008;1(3):274-82.

5. Antonio F. Hernandez, Department of Legal Medicine and Toxicology. University of Granada School of Medicine, Environmental Research 2006;102:70-6.

6. Weissmann-Brenner A, Aviv-Vidan A, Hourvitz A. Organophosphate poisoning: a multihospital survey. IMAJ 2002;4:573-6.

7. Sniderman AD. Is there value in liver function test and creatine phosphokinase monitoring with statin use? Am J Cardiol 2004;94(9A):30F-4.

8. Perreault S, Birca A, Piper B, et al. Transient creatine phosphokinase elevations in children: a single-centre experience. J Paediatr 2011;159(4):682-5. 